TRANSACTIONS OF THE

AMERICAN MATHEMATICAL SOCIETY

Volume 275, Number 2, February 1983

\title{
A GENERAL MAXIMAL OPERATOR AND THE $A_{p}$-CONDITION \\ BY
}

\author{
M. A. LECKBAND AND C. J. NEUGEBAUER
}

\begin{abstract}
A rearrangement inequality for a general maximal operator $M f(x)=$ $\sup _{x \in Q} \int f \phi_{Q} d \nu$ is established. This is then applied to the Hardy-Littlewood maximal operator with weights.
\end{abstract}

1. Let $\mu, \nu$ be two measures on $\mathbf{R}^{n}$ and let there be given for each cube $Q \subset \mathbf{R}^{n}$ a function $\phi_{Q}$ supported in $Q$. We consider the maximal operator $M f(x)=\sup \int f \phi_{Q} d \nu$, where the sup is extended over all cubes centered at $x$ and obtain (Theorem 1) the rearrangement inequality $(M f)_{\mu}^{*}(\xi) \leqslant A \int_{0}^{\infty} \Phi(t) f_{\nu}^{*}(t \xi) d t$. Here $g_{\lambda}^{*}$ denotes the nonincreasing rearrangement of $g$ with respect to the measure $\lambda$, and $\Phi$ is a nonincreasing function given in terms of $\mu, \nu, \phi_{Q}$. From this one easily sees that $\|M f\|_{p, \mu} \leqslant$ $A\|f\|_{p, \nu} \int_{0}^{\infty} \Phi(t) / t^{1 / p} d t$, and thus the finiteness of this integral, i.e., $\Phi \in L\left(p^{\prime}, 1\right)$, gives a weighted norm inequality. This is how the $A_{p}$-condition comes into play. In fact, if we take $(u, v) \in A_{p}$, i.e., $\int_{Q} u \cdot\left(\int_{Q} v^{1-p^{\prime}}\right)^{p-1} \leqslant C|Q|^{p}$ [5], $d \mu=u d x, d v=$ $v d x, \phi_{Q}(x)=\chi_{Q}(x) /|Q| v(x)$, then the above $M f(x)$ is the usual Hardy-Littlewood maximal operator. Let $\Phi=\Phi_{u, v}$ be the associated $\Phi$. We will show (Theorem 3) that, in the case $u=v, \Phi \in L\left(p^{\prime}, 1\right)$ if and only if $u \in A_{p}$, and in the double weight situation (Theorem 4), $\Phi \in L\left(p^{\prime}, \infty\right)$ if and only if there is $(\bar{u}, \bar{v})$ for which $\Phi_{\bar{u}, \bar{v}} \sim \Phi$ and $\|M f\|_{p, \bar{u}} \leqslant A\|f\|_{p, \bar{v}}$.

Finally, we will study the problem when $(u, v) \in A_{p}$ implies $\|M f\|_{p, u} \leqslant A\|f\|_{p, v}$, and the extrapolation problem, i.e., when does $\|M f\|_{p . u} \leqslant A\|f\|_{p, v}$ imply the existence of $\varepsilon>0$ so that $\|M f\|_{p-\varepsilon, u} \leqslant B\|f\|_{p-\varepsilon, v}$ ? It turns out that the behavior of the iterated maximal operator $M_{j}$ is crucial here. We will see (Theorem 6) that extrapolation is possible provided the norm of $M_{j}$ as an operator from $L_{v}^{p} \rightarrow L_{u}^{p}$ grows at most geometrically, a fact which is obvious for $u=v$. All this gives a different, though admittedly long, proof of $u \in A_{p}$, implies $u \in A_{p-\varepsilon}$, and shows that it is the iterated maximal operator that controls this implication.

2. For $\nu \geqslant 0$ a Borel measure on $\mathbf{R}^{n}$ and $f: \mathbf{R}^{n} \rightarrow \mathbf{R}$ a Borel measurable function, let $\lambda_{f, \nu}(y)=\nu\{x:|f(x)|>y\}$, and $f_{\nu}^{*}(t)=\inf \left\{y: \lambda_{f, \nu}(y) \leqslant t\right\}$, the rearrangement of $f$ with respect to $\nu$. With each $Q \in\{Q\}$, the collection of cubes in $\mathbf{R}^{n}$, let there be associated a Borel measurable function $\phi_{Q}: \mathbf{R}^{n} \rightarrow[0, \infty)$, supp $\phi_{Q} \subset Q$. We consider the general maximal operator.

$$
M f(x)=\sup \int_{\mathbf{R}^{n}} \phi_{Q} f d \nu
$$

Received by the editors August 4, 1981 and, in revised form, February 17, 1982.

1980 Mathematics Subject Classification. Primary 42A96; Secondary 28A15. 
where the sup is extended over all $Q$ with center $x$. If $\mu \geqslant 0$ is another Borel measure on $\mathbf{R}^{n}$, finite on compact sets, define

$$
\Phi(t)=\sup _{Q}\left\{\mu(Q) \phi_{Q, \nu}^{*}(\mu(Q) t)\right\} .
$$

THEOREM 1.

$$
(M f)_{\mu}^{*}(\xi) \leqslant A \int_{0}^{\infty} \Phi(t) f_{\nu}^{*}(t \xi) d t
$$

where $A$ depends only upon the dimension $n$.

Proof. We let $M_{r} f(x)=\sup \int \phi_{Q} f d \nu$, where now the sup is extended over all $Q$ with center $x$ and diam $Q \leqslant r$. It suffices to prove the theorem for $M_{r} f$ and then let $r \rightarrow \infty$.

Let $E_{\tau}=\left\{x: M_{r} f(x)>\tau\right\}$ and $E_{\tau, R}=E_{\tau} \cap\{|x| \leqslant R\}$. For $x \in E_{\tau, R}$, we have a cube $Q_{x}$, center $x$, diam $Q_{x} \leqslant r$ such that $\tau \leqslant \int \phi_{Q_{x}} f d \nu$. We can now apply the Besicovitch covering theorem [1] and select $\left\{Q_{j}\right\} \subset\left\{Q_{x}: x \in E_{\tau, R}\right\}$ such that $E_{\tau, R} \subset \cup Q_{j}$ and $\Sigma \chi_{Q_{j}}(t) \leqslant C$, where $C$ depends only upon $n$. Then $\mu\left(Q_{j}\right) \tau \leqslant$ $\int \mu\left(Q_{j}\right) \phi_{Q_{1}} f d \nu$. We set $H_{N}=\sum_{j=1}^{N} \mu\left(Q_{j}\right), \Phi_{N}(y)=\sum_{j=1}^{N} \mu\left(Q_{j}\right) \phi_{Q_{j}}(y)$. Then

$$
H_{N} \leqslant \frac{1}{\tau} \int_{\mathbf{R}^{n}} \Phi_{N}(y) f(y) d \nu \leqslant \frac{1}{\tau} \int_{0}^{\infty} \Phi_{N, \nu}^{*}(t) f_{\nu}^{*}(t) d t .
$$

We claim now that $\Phi_{N, \nu}^{*}(\xi) \leqslant c \Phi\left(\xi / H_{N}\right)$, where $c$ is the Besicovitch constant. If $\Phi_{N}(y)>\alpha, \alpha>0$, then $y \in \cup_{j=1}^{N} Q_{j}$. Thus the number of $Q_{j}$ 's containing $y$ is at most $c$, and hence for some $j, \mu\left(Q_{j}\right) \phi_{Q_{i}}(y)>\alpha / c$. Thus

$$
\left\{y: \Phi_{N}(y)>\alpha\right\} \subset \bigcup_{j=1}^{N}\left\{y: \mu\left(Q_{j}\right) \phi_{Q_{j}}(y)>\alpha / c\right\} .
$$

We now show that for $\beta>0$,

$$
\nu\left\{y: \mu(Q) \phi_{Q}(y)>\beta\right\} \leqslant \mu(Q)|\{t: \Phi(t)>\beta\}| .
$$

To prove this we may assume that $\mu(Q)>0$. Then

$$
\begin{aligned}
|\{t: \Phi(t)>\beta\}| & \geqslant\left|\left\{t: \mu(Q) \phi_{Q, \nu}^{*}(\mu(Q) t)>\beta\right\}\right| \\
& =\frac{1}{\mu(Q)} \nu\left\{y: \mu(Q) \phi_{Q}(y)>\beta\right\} .
\end{aligned}
$$

All this gives us $\nu\left\{y: \Phi_{N}(y)>\alpha\right\} \leqslant H_{N}|\{t: \Phi(t)>\alpha / c\}|$. Consequently, $\Phi_{N, \nu}^{*}(\xi)$ $\leqslant \inf \left\{\alpha:|\{t: \Phi(t)>\alpha / c\}| \leqslant \xi / H_{N}\right\}=c \Phi\left(\xi / H_{N}\right)$.

Thus

$$
\tau \leqslant \frac{c}{H_{N}} \int_{0}^{\infty} \Phi\left(\frac{t}{H_{N}}\right) f_{\nu}^{*}(t) d t \leqslant \frac{c}{H_{N}} \int_{0}^{\infty} \Phi\left(\frac{t}{H}\right) f_{\nu}^{*}(t) d t
$$

since $H_{N} \leqslant H$, and $H=\Sigma \mu\left(Q_{j}\right)<\infty$. Since $H_{N} \uparrow H$, we get

$$
\tau \leqslant \frac{c}{H} \int_{0}^{\infty} \Phi\left(\frac{t}{H}\right) f_{\nu}^{*}(t) d t=c \int_{0}^{\infty} \Phi(t) f_{\nu}^{*}(t H) d t
$$


and since $\mu\left(E_{\tau, R}\right) \leqslant H$, we see that $\tau \leqslant c \int_{0}^{\infty} \Phi(t) f_{\nu}^{*}\left(t \mu\left(E_{\tau, R}\right)\right) d t$. Finally, let $\tau_{0}=$ $\left(M_{r} f\right)_{\mu}^{*}(\xi)=\inf \left\{\tau: \mu\left(E_{\tau}\right) \leqslant \xi\right\}$. Then $0<\tau<\tau_{0}$ implies that $\mu\left(E_{\tau}\right)>\xi$, and hence for some $R, \mu\left(E_{\tau, R}\right)>\xi$. From this we get $\tau \leqslant c \int_{0}^{\infty} \Phi(t) f_{\nu}^{*}(t \xi) d t$, and letting $\tau \uparrow \tau_{0}$, completes the proof.

REMARK. Theorem 1 contains many of the known maximal inequalities.

(i) The choice $\phi_{Q}(y)=\chi_{Q}(y) /|Q|, \mu=\nu=$ Lebesgue measure, gives the ordinary Hardy-Littlewood maximal function. In this case $\Phi(t)=\chi_{[0,1]}(t)$ and so $(M f)^{*}(\xi) \leqslant A \int_{0}^{1} f^{*}(t \xi) d t$.

(ii) Let $Q_{0}$ be the unit cube centered at the origin, and let $Q(x, h)$ be the cube with center $x$, side-length $h$. Let supp $\phi \subset Q_{0}$, and set $\phi_{Q}(y)=\phi((x-y) / h) / h^{n}, Q=$ $Q(x, h)$. If $\mu=\nu=$ Lebesgue measure, we consider the maximal "approximate identity" operator $M f(x)=\sup _{h>0}\left(1 / h^{n}\right) \int \phi((x-y) / h) f(y) d y$ [4]. In this case $\lambda_{\phi_{Q}}(y)=|Q||\{x: \phi(x)>y|Q|\}|$, and hence $\phi_{Q}^{*}(t)=\phi^{*}(t /|Q|) /|Q|$. Thus $\Phi(t)$ $=\phi^{*}(t)$, and we get $(M f)^{*}(\xi) \leqslant A \int_{0}^{1} \phi^{*}(t) f^{*}(t \xi) d t$. This maximal inequality is due to Jurkat and Troutman [4] and our proof of Theorem 1 is a refinement of theirs.

3. Minkowski's integral inequality and Theorem 1 show that

$$
\|M f\|_{p, \mu} \leqslant A\left(\int_{0}^{\infty} \frac{\Phi(t)}{t^{1 / p}} d t\right)\|f\|_{p, \nu},
$$

and hence $\int_{0}^{\infty} \Phi(t) / t^{1 / p} d t<\infty$ implies that $M f$ is strong $(p, p)$. In the setting of Lorentz spaces $L(p, q)$ [2], this says that $\Phi \in L\left(p^{\prime}, 1\right), 1 / p+1 / p^{\prime}=1$, implies strong $(p, p)$ for $M f$. A major part of this paper is devoted to the converse, i.e., when does strong $(p, p)$ for $M f$ imply $\Phi \in L\left(p^{\prime}, 1\right)$ ? Simple examples show that this need not be the case in general. For, if we consider the "approximate identity" example of the previous section and assume that $\phi$ is radially nonincreasing, then $M f(x) \leqslant\|\phi\|_{1} M_{0} f(x)$, where $M_{0}$ is the ordinary Hardy-Littlewood maximal operator. Simply take $\phi \in L^{1}, \phi \notin L\left(p^{\prime}, 1\right)$ to obtain an example.

We let now $(u, v)$ be a pair of nonnegative functions (weights), i.e., $u \in L_{\text {loc }}^{1}$ and $0<v<\infty$, a.e. $x$. This last restriction is made in order to avoid the special cases arising from division by zero, etc. Then

$$
\frac{1}{|Q|} \int_{Q} f d x=\frac{1}{|Q|} \int f \cdot \frac{\chi_{Q}}{v} v d x=\int f \cdot \phi_{Q} d \nu
$$

where $\phi_{Q}(x)=\chi_{Q}(x) /|Q| v(x)$, and $d \nu=v d x$. If we let $d \mu=u d x$ and

$$
\Phi(t) \equiv \Phi_{u, v}(t)=\sup \left\{\mu(Q) \phi_{Q, \nu}^{*}(\mu(Q) t)\right\},
$$

then $\Phi \in L\left(p^{\prime}, 1\right)$ gives the double weight strong $(p, p)$ for the ordinary HardyLittlewood maximal operator, which from now on we will denote by $M f$.

4. The single weight problem, i.e., $u=v$, and the double weight problem are different and the $\Phi$ reflects this.

THEOREM 2. Let $1<p<\infty$ and $\|M f\|_{p, u} \leqslant A\|f\|_{p, v}$. Then $\Phi=\Phi_{u, v}$ satisfies (i) $\Phi(t)=O\left(t^{-1 / p^{\prime}}\right)$, as $t \rightarrow 0$ or $\infty$, (ii) $\Phi(t)=O\left(t^{a}\right)$ for $0>a>-1$ as $t \rightarrow \infty$. 

that

Proof. It is known that $(u, v) \in A_{p}$, i.e, $\int_{Q} u \cdot\left(\int_{Q} v^{1-p^{\prime}}\right)^{p-1} \leqslant c|Q|^{p}[5]$. We note

$$
\begin{aligned}
\left(\frac{\chi_{Q}}{v}\right)_{\nu}^{*}(\mu(Q) t) & \leqslant\left[\frac{1}{\mu(Q) t} \int_{0}^{\mu(Q) t}\left(\frac{\chi_{Q}}{v}\right)_{\nu}^{* p^{\prime}}(u) d u\right]^{1 / p^{\prime}} \\
& \leqslant\left[\frac{1}{\mu(Q) t} \int_{Q}\left(\frac{1}{v}\right)^{p^{\prime}} v d x\right]^{1 / p^{\prime}}
\end{aligned}
$$

From this we get

$$
\frac{\mu(Q)}{|Q|}\left(\frac{\chi_{Q}}{v}\right)_{\nu}^{*}(\mu(Q) t) \leqslant \frac{1}{t^{1 / p^{\prime}}} \frac{\mu(Q)^{1 / p}}{|Q|} \cdot\left(\int_{Q} v^{1-p^{\prime}}\right)^{(p-1) / p} \leqslant \frac{c}{t^{1 / p^{\prime}}},
$$

and this proves (i).

For (ii) simply note that $\|M f\|_{q, u} \leqslant A_{q}\|f\|_{q, v}, p \leqslant q$, so that by (i), $\Phi(t)=$ $O\left(t^{-1 / q^{\prime}}\right)$.

REMARK. (i) The above result shows that the behavior of $\Phi$ about 0 is much more critical than that about $\infty$. (ii) We have shown that $(u, v) \in A_{p}$ implies that $\Phi \in L\left(p^{\prime}, \infty\right)$.

THEOREM 3. $\|M f\|_{p, u} \leqslant A\|f\|_{p, u}$ for some $p>1$ if and only if $\Phi \in L\left(p^{\prime}, 1\right)$, i.e., $\Phi \in L\left(p^{\prime}, 1\right)$ and $u \in A_{p}$ are equivalent.

Proof. Note that now $\phi_{Q}(x)=\chi_{Q}(x) /|Q| u(x)$, so that $\phi_{Q, \mu}^{*}(\mu(Q) t)$ is zero for $t>1$, and hence $\Phi(t)=0, t>1$. Thus we have to show that $\int_{0}^{1} \Phi(t) / t^{1 / p} d t<\infty$. Since $u \in A_{p-\varepsilon}$ for some $\varepsilon>0$ [5], we get from Theorem 2 that $\Phi \in L\left((p-\varepsilon)^{\prime}, \infty\right)$ from which $\Phi(t) / t^{1 / p} \leqslant c / t^{1 /(p-\varepsilon)^{\prime}+1 / p}$.

ReMarK. Later we will show that $\Phi \in L\left(p^{\prime}, 1\right)$ implies $\Phi \in L\left((p-\varepsilon)^{\prime}, 1\right)$ in the single weight case without recourse to $u \in A_{p-\varepsilon}$.

5. From now on we assume that $n=1$, and we will denote by $I, J$ intervals in $\mathbf{R}$. In this section we will present a partial converse of Theorem 2, i.e., we ask whether $\Phi \in L\left(p^{\prime}, \infty\right)$ implies some norm inequality for $M f$.

For $\Phi_{1}, \Phi_{2}$ two nonincreasing functions on $(0, \infty)$ we write $\Phi_{1} \sim \Phi_{2}$ provided there are constants $c_{i}, c_{i}^{\prime}, i=1,2$, such that $c_{1} \Phi_{1}\left(c_{1}^{\prime} t\right) \leqslant \Phi_{2}(t) \leqslant c_{2} \Phi_{1}\left(c_{2}^{\prime} t\right), 0<t \leqslant 1$.

THEOREM 4. Let $\Phi_{0} \geqslant 0$ be nonincreasing on $(0, \infty)$ such that $t \Phi_{0}(t) \downarrow 0$ as $t \downarrow 0$. Then $\Phi_{0} \in L\left(p^{\prime}, \infty\right)$ on $[0,1]$ if and only if there exists a pair of weights $(u, v)$ such that $\Phi_{u, v} \sim \Phi_{0}$ and $\|M f\|_{p, u} \leqslant A\|f\|_{p, v}$.

REMARK. The condition $t \Phi_{0}(t) \downarrow 0$ as $t \downarrow 0$ can always be achieved by replacing $\Phi_{0}$ by $\bar{\Phi}(t)=(1 / t) \int_{0}^{t} \Phi_{0} \geqslant \Phi_{0}(t)$ and $\bar{\Phi}$ is in the same $(p>1)$ integrability class as $\Phi_{0}$.

Proof. By Theorem 2 we only need to show that $\Phi_{0} \in L\left(p^{\prime}, \infty\right)$ implies the existence of $(u, v)$. We may assume that $\Phi_{0}(1)=1$ and $\Phi_{0}(t) \uparrow \infty$ as $t \downarrow 0$ (otherwise let $u=v=1)$. Let $\alpha_{N}=\Phi_{0}\left(2^{-N}\right)$. Then $\alpha_{N} \leqslant A 2^{N / p^{\prime}}$, and since $2^{-N} \alpha_{N} \rightarrow 0$, we may assume that $\alpha_{N} \cdot 2^{-N} \leqslant \frac{1}{4}, N=1,2, \ldots$ Also note that $2^{-k} \alpha_{k+l} \leqslant \alpha_{l}$.

Let $J_{N}=\left[2^{N^{2}}, 2^{N^{2}}+\alpha_{N} 2^{-N}\right], \quad N=1,2, \ldots, \quad J_{0}=\mathbf{R} \backslash \cup_{N=1}^{\infty} J_{N}, \quad K_{N}=\left[2^{N^{2}}\right.$ $\left.+\frac{3}{4}, 2^{N^{2}}+1\right]$. Define $v_{N}(t)=\alpha_{N}^{-1}, t \in J_{N}$, and $v_{N}(t)=0, t \notin J_{N}$. Let $u_{N}(t)=4$, 
$t \in K_{N}$, and $u_{N}(t)=0, t \notin K_{N}$. The desired pair of weights will be $v(t)=\Sigma v_{N}(t)$ $+4 \chi_{J_{0}}(t), u(t)=\Sigma u_{N}(t)$. We note that $\nu\left(J_{N}\right)=2^{-N}$, from which $\left(\chi_{J_{N}}\right)_{\nu}^{*}(t)=$ $\chi_{\left[0,2^{-N}\right]}(t)$. Also $\mu\left(K_{N}\right)=1$.

We wish to estimate

$$
\Phi(t) \equiv \Phi_{u, v}(t)=\sup _{I} \frac{\mu(I)}{|I|}\left(\frac{\chi_{I}}{v}\right)_{\nu}^{*}(\mu(I) t),
$$

and show that $\Phi \sim \Phi_{0}$. This will follow if for some constants $c^{\prime}, c^{\prime \prime}, c^{\prime} \alpha_{l} \leqslant \Phi\left(2^{-l}\right) \leqslant$ $c^{\prime \prime} \alpha_{l}, l=1,2, \ldots$ Our first observation is that

$$
\left(\frac{\chi_{I}}{v}\right)_{\nu}^{*}\left(2^{-l}\right) \leqslant c \alpha_{l}, \quad l=1,2, \ldots
$$

To see this, note that if $I \cap J_{N}=\varnothing$ for every $N$, then $\left(\chi_{I} / v\right)_{v}^{*}(t) \leqslant \frac{1}{4} \leqslant \alpha_{l}$. Otherwise, let $J_{N}, J_{N+1}, \ldots, J_{M}$ be all the $J_{i}$ 's with $J_{i} \cap I \neq \varnothing$. If $I_{0}=\left[0,2^{-M}\right]$, and for $j \geqslant 1, I_{j}=\left[2^{-M}+2^{-M+1}+\cdots+2^{-M+j-1}, 2^{-M}+2^{-M+1}+\cdots+2^{-M+j}\right]$, then $\left(\chi_{I} / v\right)_{v}^{*}(t) \leqslant \alpha_{M-j}, t \in I_{j}$. Thus, if $2^{-l} \in I_{j}, M-j \leqslant l+1$, and since $2^{-N} \alpha_{N} \downarrow 0$, $2 \alpha_{l} \geqslant \alpha_{l+1} \geqslant \alpha_{M-j}$.

It is clear that $\mu(I) \leqslant 4|I|$, and if $\mu(I)>0$ and $I \cap J_{N} \neq \varnothing$ for some $N$, then $|I| \geqslant \frac{3}{4}-\alpha_{N} / 2^{N} \geqslant \frac{1}{2}$. From this we see that, if $\mu(I) \geqslant 1$, then

$$
\frac{\mu(I)}{|I|}\left(\frac{\chi_{I}}{V}\right)_{\nu}^{*}\left(\mu(I) 2^{-l}\right) \leqslant c\left(\frac{\chi_{I}}{v}\right)_{\nu}^{*}\left(2^{-l}\right) \leqslant c \alpha_{l},
$$

and if $1 / 2^{k+1} \leqslant \mu(I) \leqslant 1 / 2^{k}$, and $I \cap J_{N} \neq \varnothing$ for some $N$, then

$$
\frac{\mu(I)}{|I|}\left(\frac{\chi_{I}}{v}\right)_{\nu}^{*}\left(\mu(I) 2^{-l}\right) \leqslant c 2^{-k}\left(\frac{\chi_{I}}{v}\right)_{\nu}^{*}\left(2^{-k-l-1}\right) \leqslant c 2^{-k} \alpha_{k+l+1} \leqslant c \alpha_{l} .
$$

This shows that $\Phi\left(2^{-l}\right) \leqslant c \alpha_{l}$, and since for $I=\left[2^{l^{2}}, 2^{l^{2}}+1\right]$,

$$
\frac{\mu(I)}{|I|}\left(\frac{\chi_{I}}{v}\right)_{\nu}^{*}\left(\mu(I) 2^{-l}\right)=\alpha_{l}, \quad \alpha_{l} \leqslant \Phi\left(2^{-l}\right) .
$$

We proceed now with the proof of $\|M f\|_{p, u} \leqslant A\|f\|_{p, v}$. Let $f \geqslant 0, f_{N}=f \chi_{J_{N}}$, $N=0,1, \ldots$. Then

$$
\begin{aligned}
\int(M f)^{p} u d x & =\int\left[M\left(f_{0}+\sum f_{N}\right)\right]^{p} u d x \\
& \leqslant 2^{p-1} \int\left(M f_{0}\right)^{p} u d x+2^{p-1} \int\left[M\left(\sum f_{N}\right)\right]^{p} u d x .
\end{aligned}
$$

Note that $\int\left(M f_{0}\right)^{p} u d x \leqslant c \int\left(M f_{0}\right)^{p} d x \leqslant c\left\|f_{0}\right\|_{p}^{p} \leqslant c\|f\|_{p, v}^{p}$.

We next claim that $\int\left(M f_{N}\right)^{p} u_{N} d x \leqslant A \int f_{N}^{p} v_{N} d x$. For $x \in K_{N}=\operatorname{supp} u_{N}$ we have

$$
\left(M f_{N}\right)^{p}(x) \leqslant\left(2 \int_{2^{N^{2}}}^{2^{N^{2}}+\alpha_{N} 2^{-N}} f_{N} d x\right)^{p} \leqslant 2^{p}\left(\alpha_{N} 2^{-N}\right)^{p / p^{\prime}}\left\|f_{N}\right\|_{p}^{p} .
$$

Since $\alpha_{N}=O\left(2^{N / p^{\prime}}\right)$ we obtain $\left(\alpha_{N} 2^{-N}\right)^{p / p^{\prime}} \leqslant c \alpha_{N}^{-1}$ from which $\left(M f_{N}\right)^{p} \leqslant$ $c\left(1 / \alpha_{N}\right)\left\|f_{N}\right\| p=c \int f_{N}^{p} v_{N} d x$. 
We next observe that $\int M^{p}\left(\Sigma f_{N}\right) u d x=\Sigma_{k} \int M^{p}\left(\sum f_{N}\right) u_{k} d x$, and thus, using $\left(\sum a_{j}\right)^{p}$ $\leqslant \Sigma 2^{j(p-1)} a_{j}^{p}$, we get

$$
\begin{aligned}
\int M^{p}\left(\sum f_{N}\right) u_{k} d x \leqslant & 2^{p-1} \sum_{N \geqslant k} 2^{(N-k)(p-1)} \int M^{p} f_{N} u_{k} d x \\
& +2^{p-1} \sum_{N<k} 2^{(k-N)(p-1)} \int M^{p} f_{N} u_{k} d x
\end{aligned}
$$

For $k<N$ we get

$$
\begin{aligned}
\int M^{p} f_{N} u_{k} d x & \leqslant c \int\left(\frac{1}{2^{N^{2}}-2^{k^{2}}} \int_{J_{N}} f_{N}\right)^{p} u_{k}(x) d x \\
& \leqslant \frac{c}{2^{p N^{2}}}\left(\int_{J_{N}} f_{N}\right)^{p} \int u_{k} d x \leqslant \frac{c}{2^{p N^{2}}} \int M^{p} f_{N} u_{N} d x,
\end{aligned}
$$

since $\int_{\mathbf{R}} u_{k} d x=\int_{\mathbf{R}} u_{N} d x$.

Similarly, if $k>N, \int M^{p} f_{N} u_{k} d x \leqslant\left(c / 2^{p k^{2}}\right) \int M^{p} f_{N} u_{N} d x$, and thus we get

$$
\begin{aligned}
& \int M^{p}\left(\sum f_{N}\right) u_{k} d x \leqslant 2^{p-1} \int M^{p} f_{k} \cdot u_{k} d x \\
& \quad+c 2^{p-1}\left\{\sum_{N>k} \frac{2^{N(p-1)}}{2^{p N^{2}} \cdot 2^{k(p-1)}} \int M^{p} f_{N} \cdot u_{N} d x+\sum_{N<k} \frac{2^{k(p-1)}}{2^{p k^{2}} 2^{N(p-1)}} \int M^{p} f_{N} \cdot u_{N} d x\right\} .
\end{aligned}
$$

We sum this over $k$ and interchange the order of summation to get

$$
\begin{aligned}
\int M^{p} f u d x \leqslant & 2^{(p-1)} \sum_{k=1}^{\infty} \int M^{p} f_{k} u_{k} d x \\
& +c 2^{p-1}\left\{\sum_{N=1}^{\infty} \sum_{k=1}^{N} \frac{2^{N(p-1)}}{2^{p N^{2}} 2^{k(p-1)}} \int M^{p} f_{N} u_{N} d x\right. \\
& \left.\quad+\sum_{N=1}^{\infty} \sum_{k=N}^{\infty} \frac{2^{k(p-1)}}{2^{p k^{2}} \cdot 2^{N(p-1)}} \int M^{p} f_{N} u_{N} d x\right\} \\
\leqslant & A \sum_{k=1}^{\infty} \int M^{p} f_{k} u_{k} d x \leqslant A \sum_{k=1}^{\infty} \int f_{k}^{p} v_{k} d x \leqslant A \int f^{p} v d x .
\end{aligned}
$$

REmark. Under the hypothesis of Theorem $4, \Phi_{0} \in L\left(p^{\prime}, \infty\right)$ if and only if there exists $(u, v)$ for which $\mu\{x: M f(x)>y\} \leqslant c\|f\|_{p, v}^{p} / y^{p}$ and $\Phi_{u, v} \sim \Phi_{0}$.

6. In order to make a more detailed study of $\Phi=\Phi_{u, v}$ for a pair of weights $(u, v)$ we need some preliminary results. Again our analysis will take place on $\mathbf{R}$.

For $f: \mathbf{R} \rightarrow[0, \infty]$, and $I, J$ compact intervals let

$$
M_{j, I} f(x)=\sup _{x \in J \subset I} \frac{1}{|J|} \int_{J} M_{j-I, I} f(y) d y,
$$

the $j$ th iterated maximal function relative to $I$. Set $M_{j, I} f(x)=0, x \notin I . M_{0, I} f(x)=$ $f(x) \chi_{I}(x)$. 
LemMa 1. Let $f \geqslant 0$ be in $L^{1}(I), \operatorname{supp} f \subset I$, and let $g \geqslant 0$ be in $L^{1}(J)$, supp $g \subset J$, and assume $|I|=|J|$. Assume there are constants $C \geqslant 1, A \geqslant 1$ with

$$
C|\{x \in I: f(x)>\alpha / A\}| \geqslant|\{y \in J: g(y)>\alpha\}|, \quad \alpha>0 .
$$

Then there is a constant $B$ so that

$$
A C B^{j} \int_{I} M_{j, I} f \geqslant \int_{J} M_{j, J} g .
$$

Proof. We will first establish that for $\alpha>0$

$$
2 B C\left|\left\{x \in I: M_{1, I} f(x)>\frac{\alpha}{2 A}\right\}\right| \geqslant\left|\left\{y \in J: M_{1, J} g(y)>\alpha\right\}\right|,
$$

where $B$ is the Besicovitch covering constant. To do this, we may assume that $\alpha / 2 A \geqslant(1 /|I|) \int_{I} f$, as otherwise $M_{1, I} f(x)>\alpha / 2 A, x \in I$, and (1) follows. We have

$$
\left|\left\{y \in J: M_{1, J} g(y)>\alpha\right\}\right| \leqslant \frac{2 B}{\alpha} \int_{\{g>\alpha / 2\}} g=\frac{2 B}{\alpha}\left[\frac{\alpha}{2} \lambda_{g}\left(\frac{\alpha}{2}\right)+\int_{\alpha / s}^{\infty} \lambda_{g}(\tau) d \tau\right],
$$

where $\lambda_{g}(\tau)=|\{x \in J: g(x)>\tau\}|$. By hypothesis this is majorized by

$$
\begin{aligned}
\frac{2 B C}{\alpha}\left[\frac{\alpha}{2} \lambda_{f}\left(\frac{\alpha}{2 A}\right)+\int_{\alpha / 2}^{\infty} \lambda_{f}\left(\frac{\tau}{A}\right) d \tau\right] & =\frac{2 B C A}{\alpha} \int_{\{f>\alpha / 2 A\}} f \\
& \leqslant 2 B C\left|\left\{x \in I: M_{1, I} f(x)>\frac{\alpha}{2 A}\right\}\right|
\end{aligned}
$$

(see [8, p. 23]).

We now iterate (1) and get

$$
(2 B)^{j} C\left|\left\{x \in I: M_{j, I} f(x)>\frac{\alpha}{2^{j} A}\right\}\right| \geqslant\left|\left\{y \in J: M_{j, J} g(y)>\alpha\right\}\right| .
$$

Consequently, $\int_{J} M_{j, J} g=\int_{0}^{\infty} \lambda_{M_{j, J}} g(\alpha) d \alpha \leqslant(4 B)^{j} C A \int_{I} M_{j, I} f$.

LeMMA 2. Let $(u, v) \in A_{p}$ for some $p>1$, i.e., $\int_{I} u \cdot\left(\int_{I} v^{1-p^{\prime}}\right)^{p-1} \leqslant c|I|^{p}$, and form $\Phi=\Phi_{u, v}$. Assume that $\left|v^{-1}(t)\right|=0, t>0$. Then for each $N$ there exists $\alpha_{N}$ and compact intervals $I_{N} \supset J_{N}, I_{N} \supset I_{N}^{\prime}$, such that $J_{N} \cap I_{N}^{\prime}=\varnothing$ and $J_{N}, I_{N}^{\prime}$ have an endpoint in common with $I_{N}$, and there is $S_{N} \subset J_{N}$ such that

(i) $\Phi\left(2^{-N}\right) \leqslant c \mu\left(I_{N}^{\prime}\right) \alpha_{N} /\left|I_{N}\right| \leqslant c 2^{N / p^{\prime}}$,

(ii) $\alpha_{N} \leqslant 1 / v(x) \leqslant 5 \alpha_{N}, x \in S_{N}$,

(iii) $\mu\left(I_{N}^{\prime}\right) /\left(5 \cdot 2^{N}\right) \leqslant \nu\left(S_{N}\right) \leqslant \mu\left(I_{N}^{\prime}\right) / 2^{N}$,

(iv) $\alpha_{N} \leqslant\left(\chi_{S_{N}} / v\right)_{\nu}^{*}\left(\mu\left(I_{N}^{\prime}\right) /\left(5 \cdot 2^{N}\right)\right) \leqslant 5 \alpha_{N}$.

Proof. Since

$$
\Phi\left(2^{-N}\right)=\sup _{I} \frac{\mu(I)}{|I|}\left(\frac{\chi_{I}}{v}\right)_{\nu}^{*}\left(\frac{\mu(I)}{2^{N}}\right)
$$

choose an interval $\bar{I}_{N}=\left[a_{N}, b_{N}\right]$ for which

$$
\Phi\left(2^{-N}\right) \leqslant 2 \frac{\mu\left(\bar{I}_{N}\right)}{\left|\bar{I}_{N}\right|}\left(\frac{\chi_{I_{N}}}{v}\right)_{\nu}^{*}\left(\mu\left(\bar{I}_{N}\right) 2^{-N}\right) .
$$


We can pick points $a_{N}=x_{0}<x_{1}<x_{2}<x_{3}<x_{4}=b_{N}$ for which $\int_{x_{i-1}}^{x_{i}} u d x=$ $\mu\left(\bar{I}_{N}\right) / 4, i=1,2,3,4$. If $I_{N, i}=\left[x_{i-1}, x_{i}\right]$, we have, since

$$
\left(\frac{\chi_{I_{N}}}{v}\right)_{\nu}^{*}(\tau) \leqslant \sum_{i=1}^{4}\left(\frac{\chi_{I_{N, i}}}{v}\right)_{\nu}^{*}\left(\frac{\tau}{4}\right)
$$

an $i$ such that for each $j$,

$$
\Phi\left(2^{-N}\right) \leqslant c \frac{\mu\left(I_{N, j}\right)}{\left|\bar{I}_{N}\right|}\left(\frac{\chi_{I_{N, i}}}{v}\right)_{\nu}^{*}\left(\frac{\mu\left(I_{N, j}\right)}{2^{N}}\right) .
$$

Select now a $j$ so that $I_{N, j} \cap I_{N, i}=\varnothing$, and write $J_{N}=I_{N, i}, J_{N}^{*}=I_{N, j}$ and let $I_{N}$ be the smallest interval containing $J_{N} \cup J_{N}^{*}$. Then

$$
\Phi\left(2^{-N}\right) \leqslant c \frac{\mu\left(J_{N}^{*}\right)}{\left|I_{N}\right|}\left(\frac{\chi_{J_{N}}}{v}\right)_{\nu}^{*}\left(\frac{\mu\left(J_{N}^{*}\right)}{2^{N}}\right) .
$$

Let us denote by $J^{\prime}$ an interval in $J_{N}^{*}$ which has that endpoint in common with $J_{N}^{*}$ which $J_{N}^{*}$ has in common with $I_{N}$, and set

$$
\bar{\Phi}\left(2^{-N}\right)=\sup _{J^{\prime}} \frac{\mu\left(J^{\prime}\right)}{\left|I_{N}\right|}\left(\frac{\chi_{J_{N}}}{v}\right)_{\nu}^{*}\left(\frac{\mu\left(J^{\prime}\right)}{2^{N}}\right) .
$$

Select now $J$ ' for which the sup is "attained", i.e.

$$
\bar{\Phi}\left(2^{-N}\right) \leqslant 2 \frac{\mu\left(J^{\prime}\right)}{\left|I_{N}\right|}\left(\frac{\chi_{J_{N}}}{v}\right)_{\nu}^{*}\left(\frac{\mu\left(J^{\prime}\right)}{2^{N}}\right),
$$

and let $\alpha_{N}=\left(\chi_{J_{N}} / v\right)_{\nu}^{*}\left(\mu\left(J^{\prime}\right) / 2^{N}\right)$.

We define $S_{N} \in\left\{x \in J_{N}: 5 \alpha_{N} \geqslant 1 / v(x) \geqslant \alpha_{N}\right\}$, and $S_{N}^{\prime}=\left\{x \in J_{N}: 1 / v(x) \geqslant\right.$ $\left.\alpha_{N}\right\}$. Since $\left|v^{-1}(t)\right|=0, t>0$, we see that $\nu\left(S_{N}^{\prime}\right)=\nu\left\{x \in J_{N}: 1 / v(x)>\alpha_{N}\right\}=$ $\mu\left(J^{\prime}\right) / 2^{N}$.

We claim now that $\nu\left(S_{N}\right) \geqslant \frac{1}{5} \nu\left(S_{N}^{\prime}\right)$. To prove this we may assume that $\nu\left(S_{N}^{\prime}\right)>$ $\nu\left(S_{N}\right)$. If $\nu\left(S_{N}\right)<\frac{1}{5} \nu\left(S_{N}^{\prime}\right)$, then

$$
\frac{\mu\left(J^{\prime}\right)}{2^{N}} \geqslant \nu\left(S_{N}^{\prime} \backslash S_{N}\right)>\frac{4}{5} \nu\left(S_{N}^{\prime}\right)=\frac{4}{5} \frac{\mu\left(J^{\prime}\right)}{2^{N}} .
$$

We can now choose an interval $J^{\prime \prime} \subset J_{N}^{*}$ for which $\mu\left(J^{\prime \prime}\right) 2^{-N} \leqslant \nu\left(S_{N}^{\prime} \backslash S_{N}\right) \leqslant$ $\mu\left(J^{\prime \prime}\right) 2^{-N+1}$, and $J^{\prime \prime}$ is a candidate for the sup of $\bar{\Phi}$. Then $\mu\left(J^{\prime \prime}\right)>\frac{2}{5} \mu\left(J^{\prime}\right)$, and since $\left(\chi_{S_{N}^{\prime} \backslash S_{N}} / v\right)_{\nu}^{*}\left(\mu\left(J^{\prime \prime}\right) / 2^{N}\right) \geqslant 5 \alpha_{N}$ we get

$$
\bar{\Phi}\left(2^{-N}\right) \geqslant \frac{\mu\left(J^{\prime \prime}\right)}{\left|I_{N}\right|}\left(\frac{\chi_{S_{N}^{\prime} S_{N}}}{v}\right)_{\nu}^{*}\left(\frac{\mu\left(J^{\prime \prime}\right)}{2^{N}}\right)>2 \alpha_{N} \frac{\mu\left(J^{\prime}\right)}{\left|I_{N}\right|} \geqslant \bar{\Phi}\left(2^{-N}\right) .
$$

Hence $\frac{1}{5} \mu\left(J^{\prime}\right) / 2^{N} \leqslant \nu\left(S_{N}\right) \leqslant \mu\left(J^{\prime}\right) / 2^{N}$.

If we let $I_{N}^{\prime}=J^{\prime}$, the properties (ii), (iii), and (iv) of the lemma follow, and the only thing that remains is $\mu\left(I_{N}^{\prime}\right) \alpha_{N} /\left|I_{N}\right| \leqslant c 2^{N / p^{\prime}}$. This can be done by the same argument used in Theorem 2 for (i) since $(u, v) \in A_{p}$.

7. It is well known that $u \in A_{p}, p>1$, implies that $u \in A_{p-\varepsilon}$ for some $\varepsilon>0$, and that this is no longer the case for $(u, v) \in A_{p}[5,6]$. If we want $(u, v) \in A_{p-\varepsilon}$, then 
in terms of $\Phi=\Phi_{u, v}$ we need to prove that $\Phi \in L\left((p-\varepsilon)^{\prime}, 1\right)$. Here is where the behavior of the iterated maximal operator $M_{j} f$ comes into the picture.

Theorem 5. Let $(u, v) \in A_{p}, 1<p$, and let $\Phi=\Phi_{u, v}$. Assume that $\left\|M_{j} f\right\|_{p, u} \leqslant$ $A_{j}\|f\|_{p, v}, f \in L_{v}^{p}, j=1,2, \ldots$. Then there are constants $c>0, B>0$ such that for every $j, N$,

$$
\Phi\left(2^{-N}\right) \leqslant c \frac{A_{j+1}}{B^{j}}\left(\frac{j !}{N^{j}}\right) 2^{N / p^{\prime}} .
$$

Proof. We will first show that we may assume that $\left|v^{-1}(t)\right|=0, t>0$. Since our overall assumption on $v$ is $0<v<\infty$ a.e., we choose $v(x) \leqslant \bar{v}(x) \leqslant 2 v(x)$ such that $\left|\bar{v}^{-1}(t)\right|=0, t>0$. Then $(u, \bar{v}) \in A_{p}$ and $\Phi_{u, v}(t) \leqslant 2 \Phi_{u, v}(t)$.

We now choose $I_{N} \supset J_{N}, I_{N}^{\prime}, S_{N} \subset J_{N}$, and $\alpha_{N}$ as in Lemma 2. Then

$$
\alpha_{N} \approx\left(\frac{\chi_{S_{N}}}{v}\right)_{\nu}^{*}\left(\frac{\mu\left(I_{N}^{\prime}\right)}{5 \cdot 2^{N}}\right)
$$

and

(i) $\alpha_{N} \leqslant 1 / v(x) \leqslant 5 \alpha_{N}, x \in S_{N}$,

(ii) $\left|S_{N}\right| / 5 \alpha_{N} \leqslant \nu\left(S_{N}\right) \leqslant\left|S_{N}\right| / \alpha_{N}$

(iii) $\mu\left(I_{N}^{\prime}\right) /\left(5 \cdot 2^{N}\right) \leqslant \nu\left(S_{N}\right) \leqslant \mu\left(I_{N}^{\prime}\right) / 2^{N}$,

(iv) $\Phi\left(2^{-N}\right) \leqslant c \mu\left(I_{N}^{\prime}\right) \alpha_{N} /\left|I_{N}\right| \leqslant c \cdot 2^{N / p^{\prime}}$.

We begin with

$$
\begin{aligned}
\int_{I_{N}^{\prime}}\left\{M_{j+1}\left(v^{1-p^{\prime}} \chi_{S_{N}}\right)\right\}^{p} u d x & \geqslant \frac{\mu\left(I_{N}^{\prime}\right)}{\left|I_{N}\right|^{p}}\left\{\int_{I_{N}} M_{j}\left(v^{1-p^{\prime}} \chi_{S_{N}}\right)\right\}^{p} \\
& \geqslant \frac{\mu\left(I_{N}^{\prime}\right)}{\left|I_{N}\right|^{p}}\left\{\int_{I_{N}} M_{j, I_{N}}\left(\alpha_{N}^{p^{\prime}-1} \chi_{S_{N}}\right)\right\}^{p} .
\end{aligned}
$$

By Lemma 1 this is

$$
\geqslant B^{j p} \frac{\alpha_{N}^{\left(p^{\prime}-1\right) p} \mu\left(I_{N}^{\prime}\right)}{\left|I_{N}\right|^{p}}\left(\int_{\left|S_{N}\right|}^{\left|I_{N}\right|} M_{j, H_{N}}\left(\chi_{\left[0,\left|S_{N}\right|\right]}\right) d x\right)^{p},
$$

where $H_{N}=\left[0,\left|I_{N}\right|\right]$.

Since for $\left|S_{N}\right| \leqslant t_{1} \leqslant\left|I_{N}\right|, M_{1, H_{N}}\left(\chi_{\left[0,\left|S_{N}\right|\right]}\right)\left(t_{1}\right) \geqslant\left|S_{N}\right| / t_{1}$ we see that

$$
\begin{aligned}
\int_{\left|S_{n}\right|}^{\left|I_{N}\right|} M_{2, H_{N}}\left(\chi_{\left[0,\left|S_{N}\right|\right]}\right) & \geqslant \int_{\left|S_{N}\right|}^{\left|I_{N}\right|} \frac{1}{t_{2}} \int_{\left|S_{N}\right|}^{t_{2}} M_{1, H_{N}}\left(\chi_{\left[0,\left|S_{N}\right|\right]}\right) d t_{1} \\
& \geqslant \int_{\left|S_{N}\right|}^{\left|I_{N}\right|} \frac{\left|S_{N}\right|}{t_{2}} \log \left(\frac{t_{2}}{\left|S_{N}\right|}\right) d t_{2}=\frac{\left|S_{N}\right|}{2} \log ^{2} \frac{\left|I_{N}\right|}{\left|S_{N}\right|} .
\end{aligned}
$$

Thus in general,

$$
\int_{I_{N}^{\prime}}\left\{M_{j+1}\left(v^{1-p^{\prime}} \chi_{S_{N}}\right)\right\}^{p} u d x \geqslant \frac{\alpha_{N}^{\left(p^{\prime}-1\right) p} \mu\left(I_{N}^{\prime}\right)}{\left|I_{N}\right|^{p}}\left|S_{N}\right|^{p}\left[\frac{B^{j} \log j\left(\frac{\left|I_{N}\right|}{\left|S_{N}\right|}\right)}{j !}\right]^{p} .
$$


Since $\left\|M_{j+1} f\right\|_{p, u} \leqslant A_{j+1}\|f\|_{p, v}$ we get with $f=v^{1-p^{\prime}} \chi_{S_{N}}$,

$$
\begin{aligned}
& \frac{\alpha_{N}^{\left(p^{\prime}-1\right) p}}{\left|I_{N}\right|^{p}} \mu\left(I_{N}^{\prime}\right)\left|S_{N}\right|^{p}\left[\frac{B^{j} \log \left(\frac{\left|I_{N}\right|}{\left|S_{N}\right|}\right)}{j !}\right]^{p} \\
& \leqslant A_{j+1}^{p} \int_{S_{N}} v^{1-p^{\prime}} d x \leqslant 5^{p^{\prime}-1} A_{j+1}^{p} \alpha_{N}^{p^{\prime}-1}\left|S_{N}\right|,
\end{aligned}
$$

or

$$
\frac{\alpha_{N} \mu\left(I_{N}^{\prime}\right)}{\left|I_{N}\right|^{p}}\left|S_{N}\right|^{p-1} \leqslant c\left[\frac{A_{j+1} \cdot j !}{B^{j} \log \left(\frac{\left|I_{N}\right|}{\left|S_{N}\right|}\right)}\right]^{p} .
$$

Since $\left|S_{N}\right| \leqslant 5 \alpha_{N} \nu\left(S_{N}\right) \leqslant 5 \alpha_{N} \mu\left(I_{N}^{\prime}\right) / 2^{N} \leqslant c\left|I_{N}\right| / 2^{N} \cdot 2^{N / p^{\prime}}=c\left|I_{N}\right| / 2^{N / p}$, we get $\left|I_{N}\right| /\left|S_{N}\right| \geqslant c 2^{N / p}$ from which

$$
\frac{\alpha_{N} \mu\left(I_{N}^{\prime}\right)}{\left|I_{N}\right|^{p}}\left[\frac{\alpha_{N}}{5} \frac{\mu\left(I_{N}^{\prime}\right)}{2^{N}}\right]^{p-1} \leqslant c\left[\frac{A_{j+1} \cdot j !}{B^{j} \log ^{j}\left(c 2^{N / p}\right)}\right]^{p} .
$$

From this we finally obtain

$$
\frac{\alpha_{N} \mu\left(I_{N}^{\prime}\right)}{\left|I_{N}\right|} \leqslant c \frac{A_{j+1} \cdot j !}{B^{j} N^{j}} \cdot 2^{N / p^{\prime}},
$$

and the proof is complete.

We can replace in Theorem 5 the strong $(p, p)$ for $M_{j} f$ by weak $(p, p)$ and obtain the same result. We state this as

Corollary. If $(u, v) \in A_{p}, \quad 1<p, \quad \Phi=\Phi_{u, v}, \quad$ and $\mu\left\{x: \quad M_{j} f(x)>y\right\} \leqslant$ $A_{j}\|f\|_{p, v}^{p} / y^{p}, j=1,2, \ldots$, then there are constants $c>0, B>0$ such that for every $j, N$,

$$
\Phi\left(2^{-N}\right) \leqslant c \frac{A_{j+1}}{B^{j}}\left(\frac{j !}{N^{j}}\right) 2^{N / p^{\prime}} .
$$

Proof. Start out exactly as in Theorem 5, and note that for $x \in I_{N}^{\prime}$,

$$
\left\{M_{j+1}\left(v^{1-p^{\prime}} \chi_{S_{N}}\right)\right\}^{p}(x) \geqslant\left\{\frac{1}{\left|I_{N}\right|^{p}} \int_{I_{N}} M_{j}\left(v^{1-p^{\prime}} \chi_{S_{N}}\right)\right\}^{p} .
$$

If we let $y^{p}$ be the right side of this inequality, then

$$
y^{p} \mu\left\{x: M_{j+1}\left(v^{1-p^{\prime}} \chi_{S_{N}}\right)>y\right\} \geqslant \frac{\mu\left(I_{N}^{\prime}\right)}{\left|I_{N}\right|^{p}}\left[\int_{I_{N}} M_{j}\left(v^{1-p^{\prime}} \chi_{S_{N}}\right)\right]^{p} .
$$

The rest of the proof is exactly as that of Theorem 5 .

TheOREM 6. Let $(u, v) \in A_{p}$ for some $p>1$, and form $\Phi=\Phi_{u, v}$.

(i) If $\mu\left\{x: M_{3} f(x)>y\right\} \leqslant A\|f\|_{p, v}^{p} / y^{p}$, then $\Phi \in L\left(p^{\prime}, 1\right)$ and hence $\|M f\|_{p, u} \leqslant$ $A\|f\|_{p, v}$ 
(ii) If $\sup _{\|f\|_{p, v}=1}\left\|M_{j} f\right\|_{p, u}=\sigma\left(A^{j}\right)$, then there exists $\varepsilon>0$ such that $\Phi \in$ $L\left((p-\varepsilon)^{\prime}, 1\right)$, and hence $\|M f\|_{p-\varepsilon, u} \leqslant A\|f\|_{p-\varepsilon, v}$.

Proof. To prove (i) we use the corollary and obtain $\Phi\left(2^{-N}\right) \leqslant c 2^{N / p^{\prime}} / N^{2}$ and so $\Sigma \Phi\left(2^{-N}\right) / 2^{N / p^{\prime}}<\infty$. Hence $\Phi \in L\left(p^{\prime}, 1\right)$.

For (ii) we use Theorem 5 and get $\Phi\left(2^{-N}\right) \leqslant c(A / N)^{j} j ! 2^{N / p^{\prime}}$, for some constant $A$. Since by Stirling's formula $j ! \sim \sqrt{2 \pi} e^{-j} j^{j+1 / 2}$, we get

$$
\Phi\left(2^{-N}\right) \leqslant c\left(\frac{A j}{e N}\right)^{j} j^{1 / 2} \cdot 2^{N / p^{\prime}}
$$

If we now let $\alpha=e / 2 A$ and $j=[\alpha N]$, then

$$
\Phi\left(2^{-N}\right) \leqslant c \frac{N^{1 / 2}}{2^{\alpha N}} 2^{N / p^{\prime}} \leqslant \frac{c}{N^{2}} 2^{N / p^{\prime}-\alpha N / 2} \leqslant \frac{c}{N^{2}} 2^{N /(p-\varepsilon)^{\prime}},
$$

for some $\varepsilon>0$. Thus $\Sigma \Phi\left(2^{-N}\right) / 2^{N /(p-\varepsilon)^{\prime}}<\infty$ and so $\Phi \in L\left((p-\varepsilon)^{\prime}, 1\right)$.

Remark. Theorem 6 provides us with a different proof of $u \in A_{p}$ implies $u \in A_{p-\varepsilon}$ for some $\varepsilon>0$. From [7,3] we know that $u \in A_{p}$ implies $\|M f\|_{p, u} \leqslant$ $A\|f\|_{p, u}$ without recourse to $A_{p-\varepsilon}$. But then $\left\|M_{j} f\right\|_{p, u} \leqslant A^{j}\|f\|_{p, u}$, and thus from (ii), $\|M f\|_{p-\varepsilon, u} \leqslant B\|f\|_{p-\varepsilon, u}$ from which we get $u \in A_{p-\varepsilon}$.

\section{REFERENCES}

1. M. de Guzmán, Real variable methods in Fourier analysis, Math. Studies, no. 46, North-Holland, Amsterdam, 1981.

2. R. A. Hunt On $L(p, q)$ spaces, Enseign. Math. (2) 12 (1966), 249-275.

3. R. A. Hunt, D. S. Kurtz and C. J. Neugebauer, $A$ note on the equivalence of $A_{p}$ and Sawyer's condition for equal weights (Proc. Zygmund Conf., Chicago Univ., 1981) (to appear).

4. W. B. Jurkat and J. L. Troutman, Maximal inequalities related to generalized a.e. continuity, Trans. Amer. Math. Soc. 252 (1979), 49-64.

5. B. Muckenhoupt, Weighted norm inequalities for the Hardy maximal function, Trans. Amer. Math. Soc. 165 (1972), 207-226.

6. B. Muckenhoupt and R. Wheeden, Two weight function norm inequalities for the Hardy-Littlewood maximal function and the Hilbert transform, Studia Math. 55 (1976), 279-294.

7. E. T. Sawyer, $A$ characterization of a two-weight norm inequality for maximal operators (preprint).

8. E. M. Stein, Singular integrals and differentiability properties of functions, Princeton Univ. Press, Princeton, N. J., 1970.

Department of Mathematics, Purdue University, West lafayette, Indiana 47907 (Current address of C. J. Neugebauer)

Current address (M. A. Leckband): Department of Mathematics, Florida International University, Miami, Florida 33199 